\title{
Multi-carbon dots and aptamer based signal amplification ratiometric fluorescence probe for protein tyrosine kinase 7 detection
}

\author{
Yunsu Ma, Yuan Wang, Yongjie Liu, Lujia Shi and Dongzhi Yang* (C)
}

\begin{abstract}
Background: Protein tyrosine kinase 7 (PTK 7) is a membrane receptor, which can be found in various kinds of cancers. In view of this, detection of PTK 7 in the peripheral circulation would be an effective way for the early diagnosis of cancer.
\end{abstract}

Results: In this work, a multi-carbon dots and aptamer-based signal amplification ratiometric fluorescence probe was developed. The fluorescence of the aptamer-modified $y$-CDs and b-CDs were respectively chosen as the detection signal and interior label. The fluorescence of $\mathrm{y}$-CDs was quenched by $\mathrm{Fe}_{3} \mathrm{O}_{4}$ and cDNA (complement to aptamer) compound without PTK 7, but recovered by the addition of PTK 7. Then, the free aptamer was cut by DNase I, which amplified the detection signal. The ratiometric fluorescence sensor for PTK 7 was established with the LOD of $0.016 \mathrm{ng} \mathrm{mL}^{-1}$.

Conclusions: Summary, a multi-carbon dots and aptamer-based signal amplification ratiometric fluorescence probe was developed for the detection of protein tyrosine kinase 7. The developed probe was applied to PTK 7 detection in MCF-7 cells and human serum with satisfying results, thus indicating that this probe has huge potential in clinical practice.

Keywords: Ratiometric fluorescence, PTK 7, Carbon dots, Signal amplification, Aptamer

\section{Background}

Cancer, as the disease with the highest mortality rate, attracts a huge amount of attention and during these pressing moments, the early diagnosis of cancer plays an important role in its therapy [1-3]. The detection of biomarkers in body fluid (like blood, urine, saliva) provide a safe and effective way for the early diagnosis of cancer [4-6]. Protein tyrosine kinase 7 (PTK 7), also known as colon cancer kinase-4, is a membrane receptor which is encoded by the PTK 7 gene, which modulates multiple Wnt pathways [7]. As stated in previous reports, PTK 7 is overexpressed in various tumours, such as in lung cancer,

*Correspondence: dongzhiy@xzhmu.edu.cn

School of Pharmacy, Xuzhou Medical University, Xuzhou 22004, Jiangsu, People's Republic of China gastric cancer, ovarian cancer and colon cancer and thus, the detection of PTK 7 in the peripheral circulation is a new way to make an early diagnosis [8]. It is essential to find a sensitive, selective, safe and simple way to detect PTK 7. Several analytical methods have been reported in previous research, including western blotting, flow cytometry, enzyme-linked immunosorbent assay, the fluorescence analysis method and electrochemistry immunoassay [9], among which the fluorescence analysis method has been widely used in various fields by virtue of its fast response time, low cost, simple usage and high sensitivity [10]. Therefore, the fluorescence method is perceived to be one of the most promising analytical tools for PTK 7 detection.

Nanomaterials have been wildly used in biomedical application [11-15]. Carbon dots (CDs) are a new kind 
of fluorescence nanomaterial that have attracted wide attention owing to their excellent properties including good photostability, easy modification, stable chemical properties, favourable biocompatibility and excellent water-solubility $[16,17]$. Some of their properties, specifically for metal ions, micromolecules and protein analysis, have meant that CDs have been used to set up biosensors [18-20]. Because of the relatively low concentration of PTK 7 in peripheral circulation, the biosensors for PTK 7 detection need to be equipped with high sensitivity and selectivity. However, despite possessing excellent optical performances, the fluorescence materials were found to be limited during the development of the PTK 7 sensor as they lacked good selectivity. Working from this base, an aptamer was introduced to develop a fluorescence probe. In addition to this, the loop amplification strategy was employed to enhance the sensitivity of that developed probe. Now, with these additions in place, the selectivity of PTK 7 detection was effectively increased.

Aptamers are DNA or RNA oligonucleotides, which are selected from random-sequence nucleic acid libraries by an exponential enrichment (SELEX) process, and can specifically bind themselves to target molecules through base pairing [21]. The advantages that aptamers have of being low-cost, and having high stability, and easy synthesis and labelling mean that aptamers are favoured when building biosensors [22-24]. Following this introduction of an aptamer, a significant improvement was seen in the selectivity of the fluorescence probe. As previously reported, an aptamer-based fluorescent platform for ultrasensitive adenosine was developed with a $60 \mathrm{PM}$ limit of detection (LOD) [25]. Furthermore, given that the aptamer is a single-stranded RNA or DNA that can be cut by the restriction enzyme, it became possible to introduce rolling circle amplification into the development of the biosensor. As Yao and his co-workers reported, a photonic crystal-assisted biochip was constructed using this rolling circle amplification to circulate microRNAs detection in serum with an LOD of $0.7 \mathrm{aM}$ [26]. The strategy of nucleic acid amplification was also successfully used in the construction of the fluorescence probe for microRNAs [27]. Additionally, in fluorescence sensors, the ratiometric fluorescence measurement can afford simultaneous recordings of two measurable signals at one excitation wavelength $[18,28]$. This is because one measurable signal plays the role of the interior label, which can then overcome the drawback that is the ease in which a single fluorescence measurement can be influenced by the detection conditions and probe concentrations $[29,30]$. Nonetheless, CDs are rarely used in ratiometric fluorescence biosensor for PTK 7 detection. Taking into account the excellent properties of CDs, it is essential to build a ratiometric fluorescence probe for the detection of PTK 7.

Herein, we aimed to develop a sensitive, selective and simple fluorescence sensor for the detection of PTK 7. Dual carbon dots, which emit blue and yellow fluorescence (b-CDs and y-CDs) and $\mathrm{Fe}_{3} \mathrm{O}_{4}$ MNPs, were chosen to build a ratiometric fluorescence probe. As designed, the fluorescence of $y$-CDs (modified by the aptamer of PTK 7) worked as the detection signal which was then quenched by the $\mathrm{Fe}_{3} \mathrm{O}_{4}$ magnetic nanoparticles (MNPs) and cDNA (complement to aptamer) compound. The fluorescence of b-CDs was the interior label, and $\mathrm{Fe}_{3} \mathrm{O}_{4}$ MNPs were used for magnetic separation. When encountering PTK 7, the fluorescence produced by the $\mathrm{y}$-CDs was recovered by separating it from $\mathrm{Fe}_{3} \mathrm{O}_{4}$ MNPs. In addition to this, the detection signal was loop amplified by adding DNase I. So, a ratiometric fluorescence sensor towards PTK 7 was established by measuring the fluorescence signals from the $y$-CDs and b-CDs. The developed probe was applied to the determination of PTK 7 in MCF-7 cells and human serum to ensure its effective application.

\section{Experimental section \\ Chemical and reagents}

Ferric trichloride, sodium acetate, citric acid monohydrate, diethylenetriamine, $N$-(3-dimethylaminopropyl)Nethylcarbodiimide hydrochloride (EDC), $\mathrm{N}$-hydroxysuccinimide (NHS), 4-aminobutyric acid (GABA) and $o$-phenylenediamine (OPD) were purchased from Sinopharm Chemical Reagent Co., Ltd. (Shanghai, China). Recombinant Protein Tyrosine Kinase 7 (PTK 7) was obtained from Wuhan USCN Business Co., Ltd. (Wuhan, China). The base sequences of the DNA oligonucleotides were provided as follows: aptamer of PTK7 (APT, ATC TAA CTG CTG CGC CGC CGG GAA AAT ACT GTA CGG TTA GA), cDNA (complementary to part of the PTK7 aptamer, TCT AAC CGT ACA GTA TTT TCC CGG CGG CG), and all of them were purchased from Shanghai Sangon Biotechnology Co., Ltd. (Shanghai, China). E. coli exonuclease I (DNase I, $>10 \mathrm{U}$ $\mu \mathrm{L}^{-1}$ ) was also provided by Shanghai Sangon Biotechnology. The purified water was prepared using a water purification system that was obtained from the Thermo Scientific technology Co., Ltd. (Shanghai, China). All other chemicals that did not have special specifications but were used in this paper were of analytical grade and used without further purification.

\section{Measurement and apparatus}

The fluorescence emission spectra shown in this paper were measured on a F4600 spectrofluorometer (Hitachi, Japan) with $380 \mathrm{~nm}$ excitation. FT-IR spectra and UV-vis 
spectra were respectively recorded on a FTIR-8400S spectrometer (Shimadzu, Japan) and WFN-203B spectrometer (Jingke, China). The transmission electron microscopic images (TEM) were measured by a JEM2100 transmission electron microscope (JEOL, Japan). The size distribution of nanoparticles was determined by the Nicomp380ZLS dynamic light scattering technique (Santa Barbara, USA).

\section{Synthesis of $\mathrm{Fe}_{3} \mathrm{O}_{4}$ magnetic nanoparticles $\left(\mathrm{Fe}_{3} \mathrm{O}_{4} \mathrm{MNPs}\right)$} $\mathrm{Fe}_{3} \mathrm{O}_{4}$ MNPs were synthesised according to previously reports [31]. Briefly, $0.54 \mathrm{~g}$ of $\mathrm{FeCl}_{3}$ and $1.44 \mathrm{~g}$ of sodium acetate were dissolved in $10 \mathrm{~mL}$ of glycol. The mixture was formed by adding the sodium acetate solution to the $\mathrm{FeCl}_{3}$ solution dropwise, while mixing it with a magnetic stirrer. Then, the mixture was moved to a Teflon-lined stainless-steel autoclave and heated at $200{ }^{\circ} \mathrm{C}$ for $8 \mathrm{~h}$. The $\mathrm{Fe}_{3} \mathrm{O}_{4}$ magnetic nanoparticles were obtained after the reacted mixture was washed with ethanol 3 times. Subsequently, $0.2 \mathrm{~g}$ of dry $\mathrm{Fe}_{3} \mathrm{O}_{4}$ magnetic nanoparticles were added to $50 \mathrm{~mL}$ of citric acid monohydrate solution $\left(0.1 \mathrm{~g} \mathrm{~mL}^{-1}\right)$ and placed under an ultrasound for $20 \mathrm{~min}$. Then, the mixture was mixed with a magnetic stirrer for $4 \mathrm{~h}$ at room temperature. The products of the reaction were washed with water and ethanol several times, dried under vacuum at $50{ }^{\circ} \mathrm{C}$, and then the carboxylate $\mathrm{Fe}_{3} \mathrm{O}_{4}$ MNPs were prepared.

\section{Synthesis of $\mathrm{Fe}_{3} \mathrm{O}_{4}$-CDNA}

First, cDNA was centrifuged at $4000 \mathrm{rpm}$ for $1 \mathrm{~min}$, and then $25 \mathrm{uL}$ of phosphate buffer (PBS, $\mathrm{pH}=7.5$ ) was added. Subsequently, cDNA $(100 \mu \mathrm{M})$ was heated at $95^{\circ} \mathrm{C}$ for $4 \mathrm{~min}$, cooled by ice bath for $4 \mathrm{~min}$, and after that, left to rest at room temperature for a while [32]. This could facilitate folding cDNA into the minimum energy structure, which is responsible for specific binding. Second, a mixture of EDC $(10 \mathrm{mg})$ and NHS $(10 \mathrm{mg})$ was added into $1 \mathrm{~mL}$ of $\mathrm{Fe}_{3} \mathrm{O}_{4}$ - $\mathrm{COOH}$ solution $\left(0.2 \mathrm{mg} \mathrm{mL}^{-1}\right)$, which was then dissolved using $\mathrm{PBS}(\mathrm{pH}=6.0)$. The mixture was stirred at $25{ }^{\circ} \mathrm{C}$ for $30 \mathrm{~min}$, and the $\mathrm{pH}$ was adjusted to 7.5. Finally, $10 \mu \mathrm{L}$ of the pre-processed cDNA $(100 \mu \mathrm{M})$ and $1 \mathrm{~mL}$ of $\mathrm{Fe}_{3} \mathrm{O}_{4}-\mathrm{COOH}$ was mixed and continuously stirred at room temperature overnight to conduct the amide linkage between the carboxyl and amino groups. $\mathrm{Fe}_{3} \mathrm{O}_{4}$-cDNA was obtained through the use of magnetic separation and washed several times to remove any unreacted cDNA. The prepared $\mathrm{Fe}_{3} \mathrm{O}_{4}$-cDNA was stored in $\mathrm{PBS}(\mathrm{pH}=7.5)$ at $4{ }^{\circ} \mathrm{C}$ for further use.

\section{Synthesis of $b-C D s$ and $y-C D s$}

The b-CDs were prepared using the one-pot hydrothermal method, as previously reported in the literature [33]. Briefly, using an ultrasonic method for a period of $10 \mathrm{~min}$,
$1.2 \mathrm{~g}$ of citric acid and $600 \mu \mathrm{L}$ of diethylenetriamine were dissolved in $20 \mathrm{~mL}$ of ultrapure water. Then, the mixture was poured into a Teflon-lined stainless-steel autoclave and heated at $200{ }^{\circ} \mathrm{C}$ for $6 \mathrm{~h}$. After having cooled to room temperature, the product was collected and dialysed. Afterwards, using the vacuum drying method, the brown powder was obtained for further characterisation and research. The $\mathrm{y}$-CDs were prepared by the same method as b-CDs [34]. We used $0.3 \mathrm{~g}$ o-PD and $0.3 \mathrm{~g}$ GABA as the carbon source, which were then dissolved in $20 \mathrm{~mL}$ of ultrapure water and sonicated for $15 \mathrm{~min}$. The mixture was moved to a Teflon-lined stainless-steel autoclave, heated at $160{ }^{\circ} \mathrm{C}$ for $6 \mathrm{~h}$, and then cooled to room temperature. The brownish yellow solution was obtained after it had been dialysed and enriched using the same method used for the preparation of b-CDs. The b-CDs and $\mathrm{y}$-CDs were stored at room temperature for further research.

\section{Synthesis of $y$-CDs-APT}

To prepare $\mathrm{y}$-CDs-APT, the carboxyl groups of $\mathrm{y}$-CDs were activated by EDC and NHS. $1 \mathrm{~mL}$-CDs $\left(0.1 \mathrm{mg} \mathrm{mL}{ }^{-1}, \mathrm{pH}=6.0\right), 10 \mathrm{mg} \mathrm{EDC}$ and $10 \mathrm{mg}$ NHS were mixed and stirred at room temperature for $30 \mathrm{~min}$, and then the $\mathrm{pH}$ of the mixture was adjusted to 7.5. In this part, APT was processed using the same method as cDNA, before then being added to $\mathrm{y}$-CDs. Then, $10 \mu \mathrm{L}$ APT was incubated with $1 \mathrm{~mL}$-CDs $(100 \mu \mathrm{M})$ at room temperature for the night to allow for the synthesis of $y$-CDs-APT. After that, the prepared y-CDs- APT was stored at $4{ }^{\circ} \mathrm{C}$ for further research.

\section{PTK 7 detection}

For the detection of PTK 7, $250 \mu \mathrm{L}$ of the prepared $\mathrm{Fe}_{3} \mathrm{O}_{4}-\mathrm{cDNA}$ and $250 \mu \mathrm{L}$ of the prepared y-CDs-APT were mixed and then incubated at $25{ }^{\circ} \mathrm{C}$ for $60 \mathrm{~min}$. The mixture was then washed 3 times using PBS by magnetic separation. Following that, $600 \mu \mathrm{L}$ variable concentrations of PTK 7, $20 \mu \mathrm{L}$ b-CDs $\left(1.00 \mathrm{ng} \mathrm{mL}^{-1}\right)$ and $10 \mu \mathrm{L}$

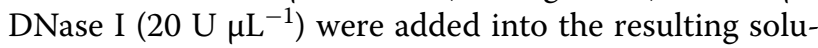
tion, and incubated at $37^{\circ} \mathrm{C}$ for $30 \mathrm{~min}$. Then, the supernatant of the resulting solution was collected through magnetic separation, and the fluorescence spectra were measured under $380 \mathrm{~nm}$ excitation.

\section{Real sample determination}

To discover the feasibility and practicality of this developed ratiometric fluorescence probe, two types of real samples (MCF-7 cells and human serum) were determined. Firstly, MCF-7 cells were incubated at $37^{\circ} \mathrm{C}$ and with $5 \%$ of $\mathrm{CO}_{2}$ for $24 \mathrm{~h}$, and then they were seeded into a plate at various densities $\left(1 \times 10^{4}, 2.5 \times 10^{4}, 5 \times 10^{4}\right.$, $1 \times 10^{5}, 2.5 \times 10^{5}$ and $5 \times 10^{5}$ per $\mathrm{mL}$ ). After $4 \mathrm{~h}$ of 
incubation, $600 \mu \mathrm{L}$ of MCF-7 cells at various densities were detected, as described in "2.7 PTK 7 detection", followed by the implementation of the standard addition method to verify the reliability of the MCF-7 cells determination. For the detection of PTK 7 in human serum samples however, the human serum was diluted with PBS by a factor of 400 . Then, the operation was carried out according to section "PTK 7 detection." The reliability of the serum sample detection was also verified using the standard addition method.

\section{Results and discussion}

\section{Materials characterisation}

The TEM images of nanoparticles are displayed in Fig. 1. As shown in Fig. 1a, the $\mathrm{Fe}_{3} \mathrm{O}_{4}$ MNPs presented a rounded shape with a diameter of about $200 \mathrm{~nm}$, and they dispersed well in the water solution [29]. The SEM images of $\mathrm{Fe}_{3} \mathrm{O}_{4}$ MNPs in Fig. 1b also displayed that they were evenly dispersed with spherical shape, which consistent with the TEM results. The TEM images of b-CDs and $y-C D$ s were shown in Fig. 1c and d, respectively [16, 35]. As shown, b-CDs were bigger, with a lattice fringe of $0.31 \mathrm{~nm}$ (insert of Fig. 1c), while y-CDs had a lattice fringe of $0.26 \mathrm{~nm}$ (insert of Fig. 1d). Both of them showed a spherical shape and were evenly dispersed when in aqueous solution.

The FT-IR spectra were measured for the purpose of characterising the structures of the nanomaterials used in this study. As shown in Fig. 2a, the broad vibration band around $3400 \mathrm{~cm}^{-1}$ was ascribed to $-\mathrm{OH}$ stretching vibration, while the peaks at 1680 and $1200 \mathrm{~cm}^{-1}$ were ascribed to $\mathrm{C}=\mathrm{O}$ and $\mathrm{C}-\mathrm{O}$ stretching vibration, which appeared in the FT-IR spectra of b-CDs, y-CDs and $\mathrm{Fe}_{3} \mathrm{O}_{4}$ MNPs [36, 37]. It can be summarised from the above stated results that there were carboxyl groups on their surface. For b-CDs and y-CDs, the FT-IR spectra also showed peaks at 1580 and $1490 \mathrm{~cm}^{-1}$, which were attributed to the $\mathrm{C}=\mathrm{C}$ vibrations in the benzene ring, indicating that there were aromatic structures in both $\mathrm{b}-\mathrm{CDs}$ and $\mathrm{y}$-CDs [22, 25]. The broad vibration band around $3300 \mathrm{~cm}^{-1}$ and the peak at $1450 \mathrm{~cm}^{-1}$ were attributed to the vibrations of $\mathrm{O}-\mathrm{H} / \mathrm{N}-\mathrm{H}$ and $\mathrm{C}-\mathrm{N}$, which thus implied an existence of $-\mathrm{NH}_{2}$ in $\mathrm{y}$-CDs [34]. For $\mathrm{Fe}_{3} \mathrm{O}_{4}$ MNPs, the stretching vibrations of $\mathrm{Fe}-\mathrm{O}$ showed a characteristic peak near $740 \mathrm{~cm}^{-1}$ in the FT-IR spectra [31].

The UV-vis absorption spectra and fluorescence spectra were measured to further study the characterisation of b-CDs and y-CDs. The UV-vis absorption spectrum of b-CDs (Fig. 2b) exhibited two obvious absorption
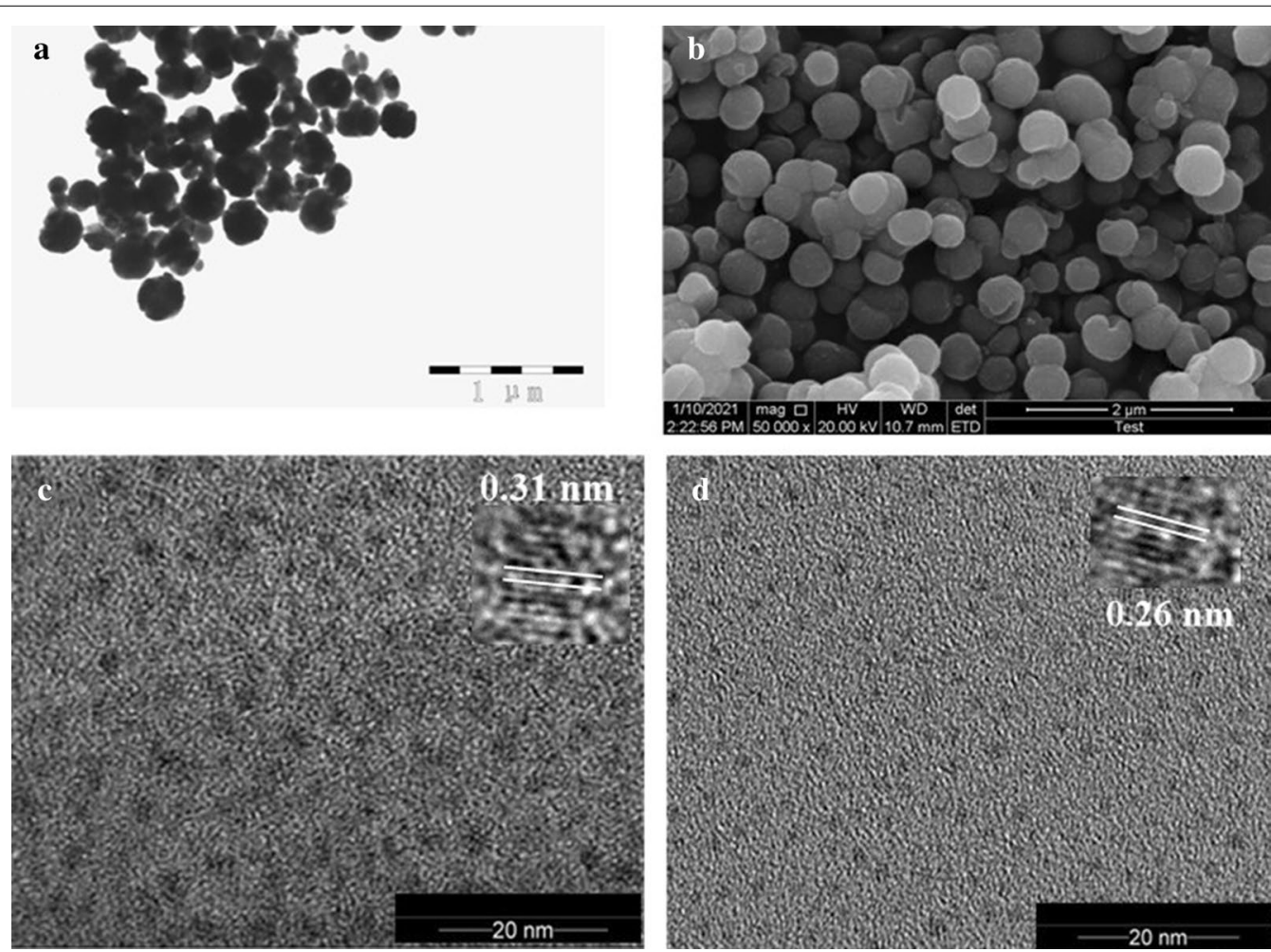

Fig. 1 a The TEM images of $\mathrm{Fe}_{3} \mathrm{O}_{4}$ MNPs. b The SEM images of $\mathrm{Fe}_{3} \mathrm{O}_{4}$ MNPs. $\mathbf{c}$ The TEM image of b-CDs, Insert: high-resolution TEM image of b-CDs. d The TEM image of $\mathrm{y}$-CDs, Insert: high-resolution TEM image of $\mathrm{y}$-CDs 

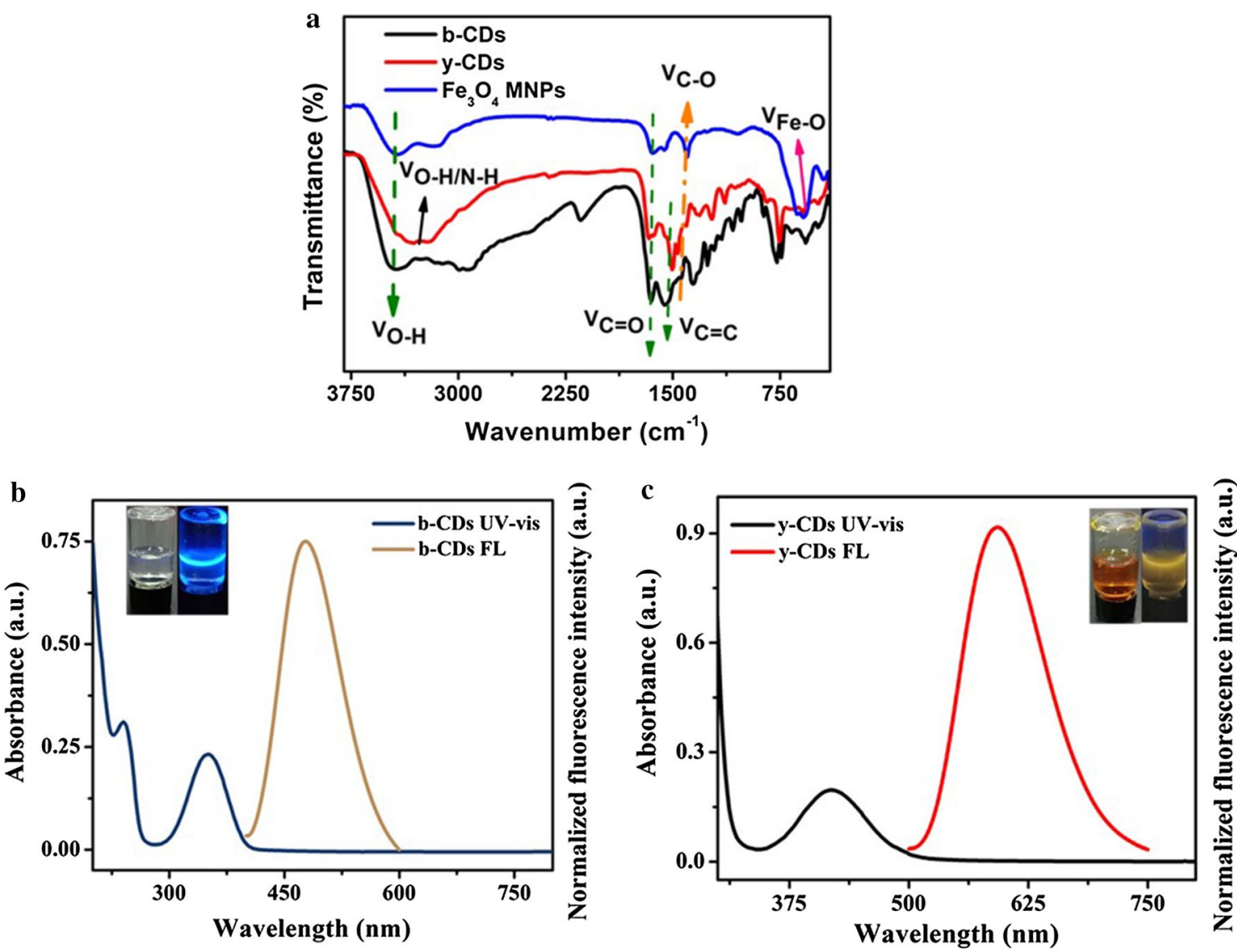

Fig. 2 a The FT-IR spectra of b-CDs, $y-C D s$ and $\mathrm{Fe}_{3} \mathrm{O}_{4}$ MNPs. b The UV-vis absorption spectrum and fluorescence spectrum of b-CDs. Insert: the solution of b-CDs under day light and UV-lamp, respectively. c The UV-Vis absorption spectrum and fluorescence spectrum of $y$-CDs. Insert: the solution of $\mathrm{y}$-CDs under day light and UV-lamp, respectively

bands at $\sim 260 \mathrm{~nm}$ and $\sim 340 \mathrm{~nm}$, which resulted from $\pi-\pi *$ transition of $\mathrm{sp}^{2}$ carbon and $\mathrm{n}-\pi^{*}$ transition of $\mathrm{C}=\mathrm{O} / \mathrm{C}=\mathrm{N}$ in $\mathrm{b}-\mathrm{CDs}$, which were in accordance with the FT-IR results $[35,38]$. As shown in Fig. $2 b$, the fluorescence spectrum of b-CDs displayed a sharp peak at $445 \mathrm{~nm}$, which was due to being excited at the wavelength of $380 \mathrm{~nm}$. The insert images in Fig. $2 \mathrm{~b}$ show the respective solution of $\mathrm{b}-\mathrm{CDs}$ under day light and UVlamp. As shown, it was colourless when under day light but had blue emissions when under the UV-lamp. The UV-vis absorption spectrum of $y$-CDs also exhibited two obvious absorption bands at $\sim 260 \mathrm{~nm}$ and $\sim 410 \mathrm{~nm}$ in Fig. 2c. The peak at $260 \mathrm{~nm}$ was considered as $\pi-\pi^{*}$ transition caused by sp 2 domains in the core of $y$-CDs, while the wide absorption band at $410 \mathrm{~nm}$ could be denoted as the functional groups, like $\mathrm{C}=\mathrm{O} / \mathrm{C}=\mathrm{N}$, which were attached to the surface of $y$-CDs $[16,34]$. The fluorescence of $\mathrm{y}$-CDs had a yellow emission, and when excited at $380 \mathrm{~nm}$ the fluorescence spectrum showed an emission wavelength at $575 \mathrm{~nm}$. In addition, the insert images in Fig. 2b showed that the solution of $y$-CDs was brown when under day light but had a yellow emission when under UV-lamp.

\section{Principle of PTK 7 sensing}

In this paper, a ratiometric fluorescence probe for the detection of PTK 7 was developed based on dual carbon dots and then it was applied to real samples to test its effectiveness. The preparation process and PTK 7 detection mechanism of this ratiometric fluorescence probe is described in Scheme 1. Firstly, cDNA (complementary to part of the PTK7 aptamer) was connected with $\mathrm{Fe}_{3} \mathrm{O}_{4}$ to form $\mathrm{Fe}_{3} \mathrm{O}_{4}$-cDNA, and APT (aptamer of PTK7) was conjugated with $\mathrm{y}$-CDs to form $\mathrm{y}$-CDs-APT. The fluorescence of $\mathrm{y}$-CDs was quenched by the specific connection between $\mathrm{Fe}_{3} \mathrm{O}_{4}$-cDNA and $\mathrm{y}$-CDs-APT (y-CDs-APTcDNA- $\mathrm{Fe}_{3} \mathrm{O}_{4}$ ). The fluorescence of $\mathrm{y}$-CDs was then recovered after adding PTK7, as $\mathrm{y}$-CDs-APT conjugated with PTK7 (y-CDs-APT-PTK7) to isolate it from $\mathrm{Fe}_{3} \mathrm{O}_{4}$-cDNA. Then, the APT of y-CDs-APT-PTK7 was cut by adding DNase I, which resulted in the freeing of PTK7. Subsequently, the $\mathrm{y}$-CDs-APT-cDNA- $\mathrm{Fe}_{3} \mathrm{O}_{4}$ was 


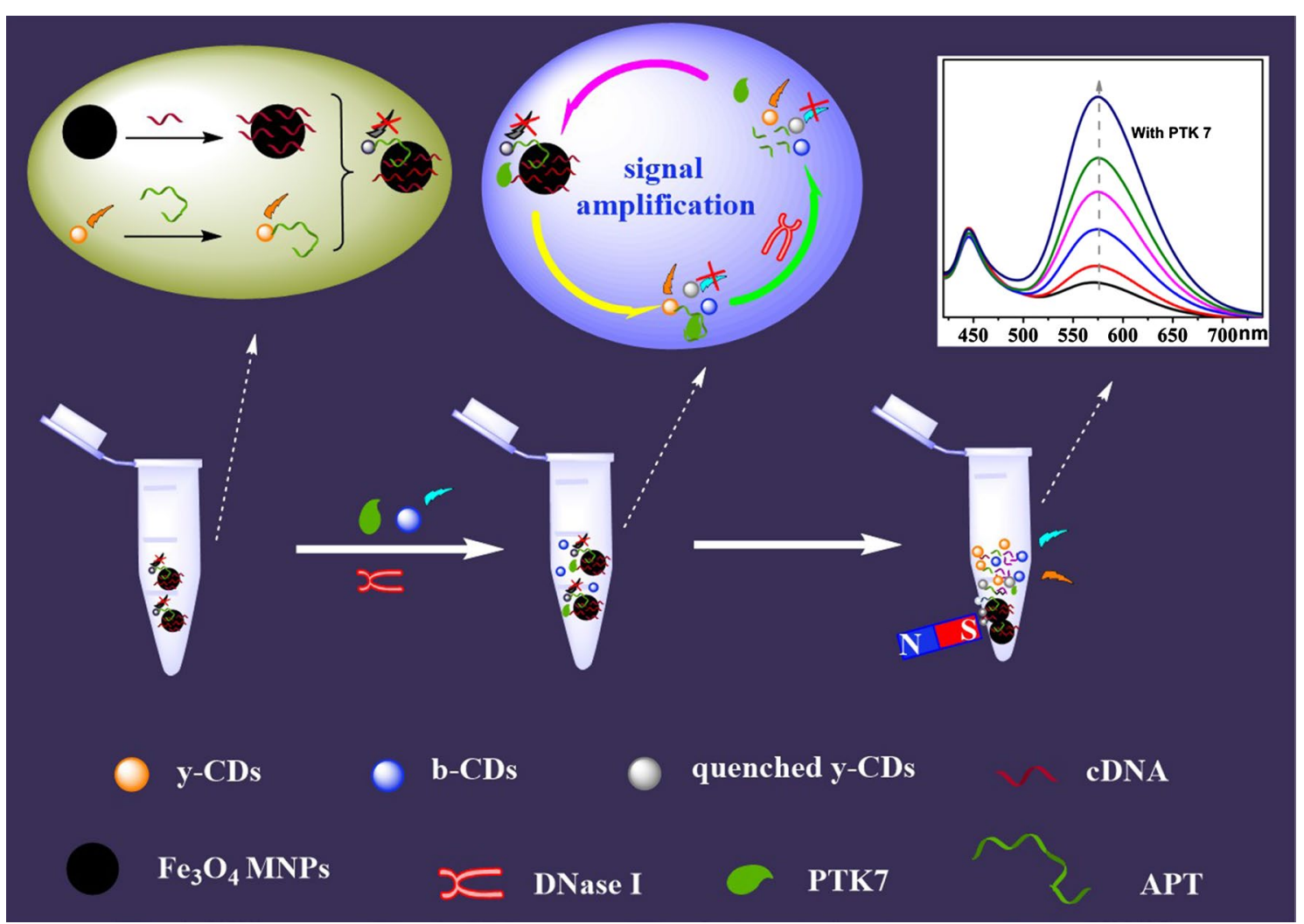

Scheme 1 Preparation process and PTK 7 detection mechanism of the ratiometric fluorescence probe

broken once more by the free PTK7, more y-CDs were removed from the surface of $\mathrm{Fe}_{3} \mathrm{O}_{4}$, and a loop amplifier was developed. In summary, the detection signal of this ratiometric fluorescence probe was provided by $y$-CDs and b-CDs, and was amplified by DNase I.

To discover a possible detection mechanism for this probe, various research papers have been studied. Obviously, the inner-filter effect based on the extensive ultraviolet absorption of $\mathrm{Fe}_{3} \mathrm{O}_{4}$ MNPs quenched the fluorescence of $y$-CDs [29]. However, the fluorescence of b-CDs with a certain concentration was quenched by $y$-CDs with increased concentrations, and the quenching degree was increasing (Fig. 3a). In addition, as shown in Fig. 3b, there was a huge overlay between the UV-vis absorption spectrum of $\mathrm{y}$-CDs and the fluorescence spectrum of b-CDs. This means that the quenching could be caused by a fluorescence resonance energy transfer or inner filter effect between b-CDs and y-CDs [39, 40]. However, the fluorescence of $\mathrm{b}-\mathrm{CDs}$ was not quenched when the probe was used to detect PTK 7. As shown in Fig. 3c, the fluorescence spectrum of b-CDs was kept at a stable value when mixed with various concentration of $y$-CDs-APT $\left(20,50,100 \mathrm{ng} \mathrm{mL}^{-1}\right)$. To discover the internal reason for whether the fluorescence of b-CDs was quenched or not, the fluorescence lifetime spectra of b-CDs, b-CDs $+\mathrm{y}$-CDs and b-CDs $+\mathrm{y}$-CDs-APT were all measured. As Fig. 3d described, when it was added to $\mathrm{y}$-CDs the fluorescence lifetime of b-CDs was reduced (from 13.78 to $1.59 \mathrm{~ns}$ ), but it showed no change after being mixed with $\mathrm{y}$-CDs-APT. The fluorescence lifetime of b-CDs meanwhile, was reduced if there was an energy transfer $[18,41]$. The data in Fig. $3 d$ indicated that whether the fluorescence quenching behaviour of b-CDs by $\mathrm{y}$-CDs disappeared or not was due to their separation. The separation of molecules in $\mathrm{y}$-CDs and b-CDs was increased by the APT modified $y$-CDs and thus, destroyed their electron or energy transfer process.

All these results stated above indicate that there is no energy or photoelectron transfer process in the sensing mechanism of this probe, which means that the sensor acts in a more controlled way.

\section{Characterisation of $\mathrm{Fe}_{3} \mathrm{O}_{4}$-cDNA and y-CDs-APT}

To build the probe, $\mathrm{Fe}_{3} \mathrm{O}_{4}$-cDNA and y-CDs-APT should be pre-composed. The conditions of this process were optimised, and the results are shown in Additional file 1: Fig. S1. As shown, when the reaction time was $60 \mathrm{~min}$ and the temperature was at $25^{\circ} \mathrm{C}$, the probe had a better 

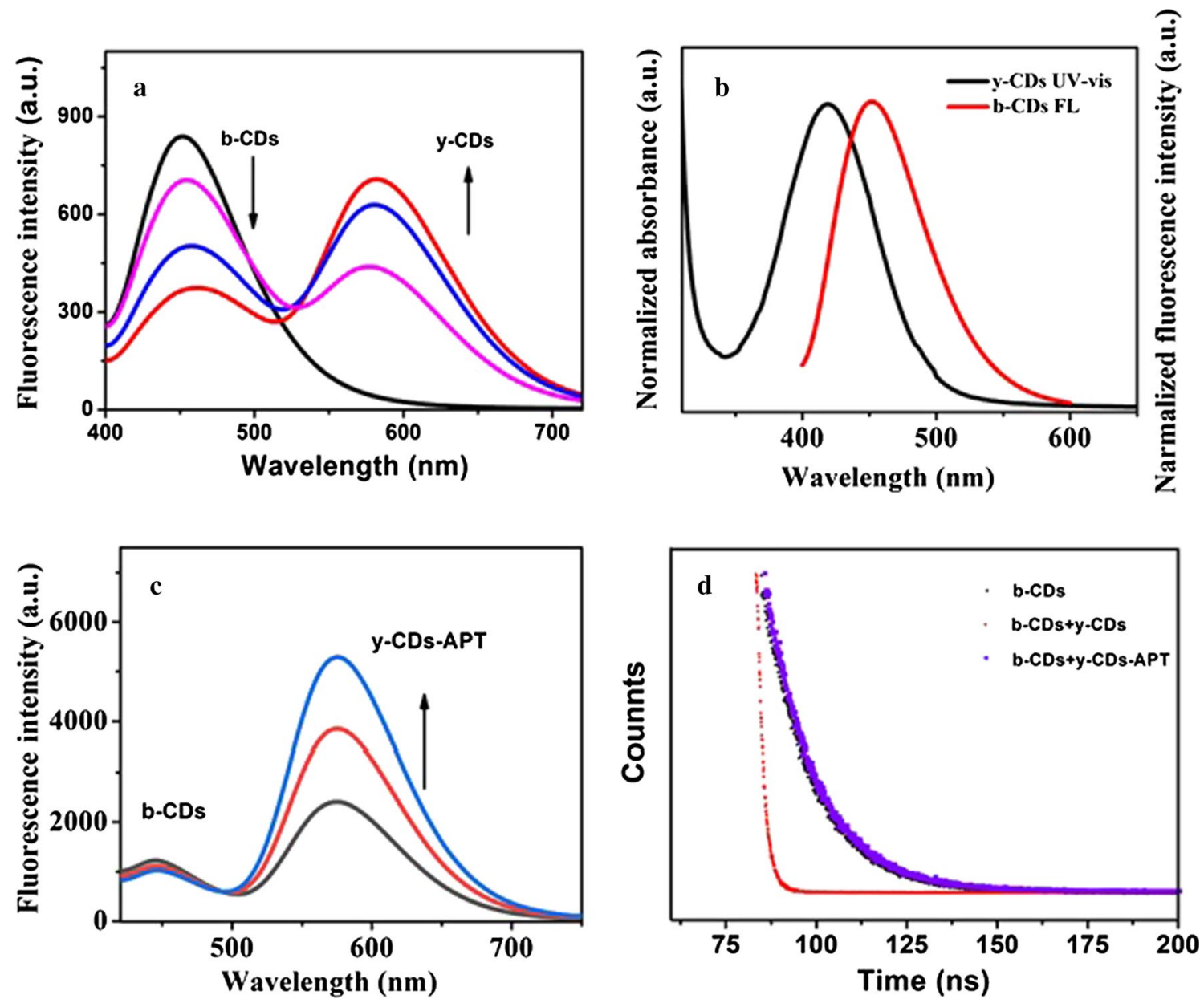

Fig. 3 a The fluorescence spectra of b-CDs mixed with various concentrations of $y$-CDs. $\mathbf{b}$ The UV-vis absorption spectrum of $y$-CDs and the fluorescence spectrum of b-CDs. c The fluorescence spectra of b-CDs mixed with various concentrations of y-CDs-APT. $\mathbf{d}$ The fluorescence lifetime spectra of b-CDs, b-CDs $+y-C D s$ and b-CDs $+y-C D s-A P T$

response to PTK 7. So, 60 min and $25{ }^{\circ} \mathrm{C}$ were chosen as the optimal temperature and reaction time, respectively. Next, to prove the connection between $\mathrm{Fe}_{3} \mathrm{O}_{4}$ MNPs and cDNA, the UV-vis absorption spectra of $\mathrm{Fe}_{3} \mathrm{O}_{4}$ MNPs, $\mathrm{Fe}_{3} \mathrm{O}_{4}-\mathrm{cDNA}$ and cDNA were shown in Fig. 4a. As shown, $\mathrm{Fe}_{3} \mathrm{O}_{4}$ MNPs showed a broad absorption band at $200 \sim 800 \mathrm{~nm}$, which was consistent with the previous report [31]. For $\mathrm{Fe}_{3} \mathrm{O}_{4}$-cDNA, a significant absorption peak was shown at $\sim 260 \mathrm{~nm}$ as well as showing the same broad absorption band as $\mathrm{Fe}_{3} \mathrm{O}_{4}$ MNPs at $200 \sim 800 \mathrm{~nm}$. Furthermore, the significant absorption peak at $\sim 260 \mathrm{~nm}$ was also shown in the UV-vis absorption spectra of cDNA [19]. The results mean a successful conjunction of $\mathrm{Fe}_{3} \mathrm{O}_{4}$ MNPs and cDNA. To prove the conjunction of APT and $y$-CDs, the AGE experiment was evaluated. As Fig. 4b shows, APT displayed no fluorescence emission when under UV lamp while the fluorescence of y-CDs and y-CDs-APT (marked by white circle) were observed.
The fluorescence spot of y-CDs-APT moved slower than that of $y$-CDs, which caused the change of shape, size and surface charge of y-CDs-APT conjugates [36]. The zeta potential of these materials was also evaluated and the results are displayed in Figure S1. The zeta potential of $\mathrm{Fe}_{3} \mathrm{O}_{4}$ MNPs and $\mathrm{y}$-CDs both show a raised change after being attached to CDNA and APT, respectively [42]. All of these results indicate the successfully connection of $\mathrm{Fe}_{3} \mathrm{O}_{4}$-cDNA and y-CDs-APT.

\section{PTK 7 determination}

To improve the sensitivity of the sensor, DNase I was added to this system, and as expected, with this addition, the developed sensor had a higher response to PTK 7. As shown in Fig. 5a, the fluorescence intensity of y-CDs (emission at $580 \mathrm{~nm}$ ) was significantly enhanced when DNase I was added, while the fluorescence intensity of b-CDs remained nearly unchanged. The adding of DNase 

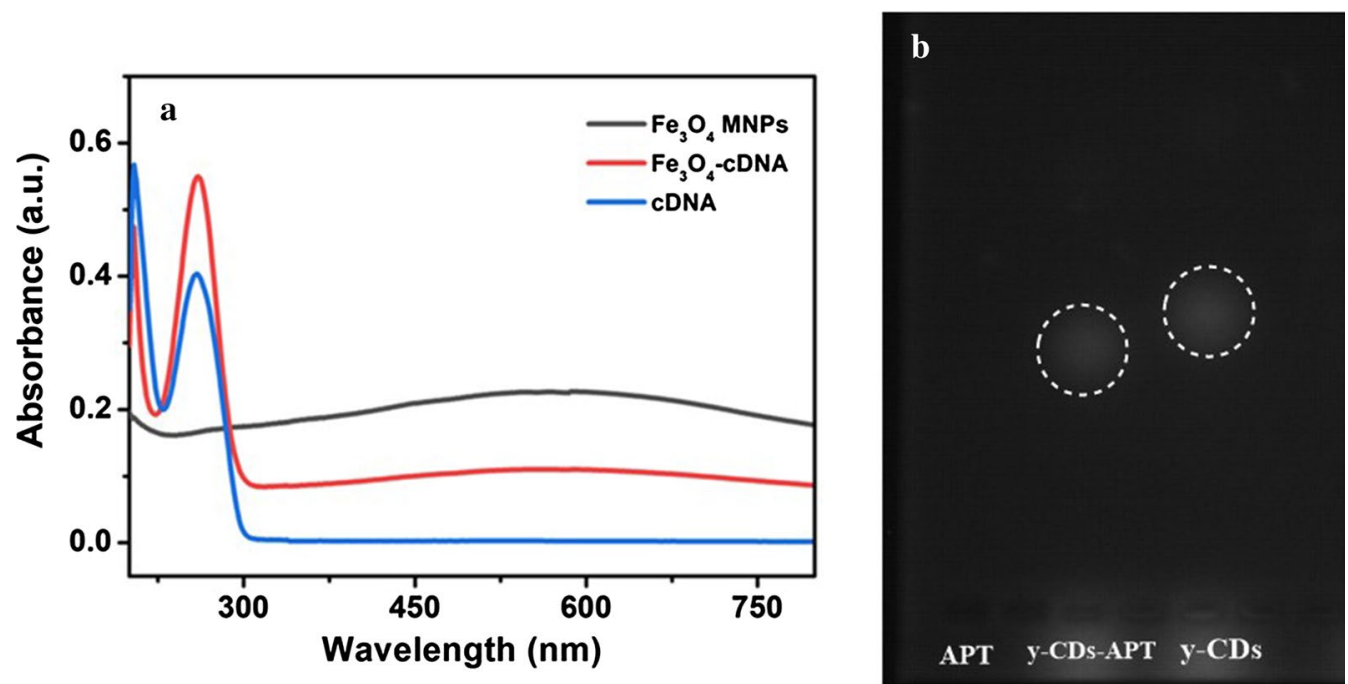

Fig. 4 a The UV-vis absorption spectra of $\mathrm{Fe}_{3} \mathrm{O}_{4} M N P S, \mathrm{Fe}_{3} \mathrm{O}_{4}-\mathrm{CDNA}$ and $C D N A$. $\mathbf{b}$ The AGE results of APT, $y$-CDs-APT and $y$-CDs
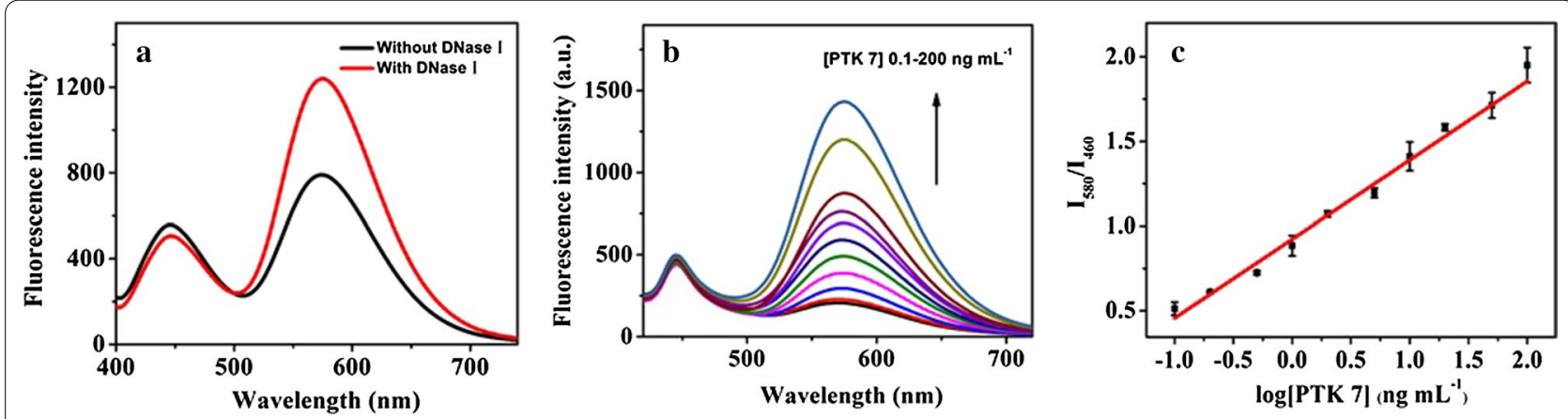

Fig. 5 a Fluorescence spectra of the sensor response to PTK 7 with or without DNase I. $\mathbf{b}$ Fluorescence spectra of the probe in the presence of various concentrations of PTK 7 (in range of $0.1-200 \mathrm{ng} \mathrm{mL}^{-1}$ ). c The calibration curve of PTK 7 detection in range of $0.1-200 \mathrm{ng} \mathrm{mL}^{-1}$

I enlarged the detection signals and improved the sensitivity of the probe. The results were consistent with the principle of this probe.

The reactive conditions were optimized through a single-factor test, and the results are displayed in Additional file 1: Fig. S2. The $\mathrm{Fe}_{3} \mathrm{O}_{4}$-cDNA and y-CDs-APT hybridisation temperature of $4{ }^{\circ} \mathrm{C}$ (Additional file 1: Fig. S2A) and 60 min reactive time were chosen as the optimum conditions. The optimum concentration (Figure S2C) and reactive time (Additional file 1: Fig. S2D) of DNase I were chosen as $20 \mathrm{U}$ and $30 \mathrm{~min}$, respectively. The changes in fluorescence intensity were then recorded under these optimised conditions and following the addition of various concentrations of PTK 7 (0, 0.1, 0.2, 0.5, 1, 2, 5, 10, 20, 50, 100 and $200 \mathrm{ng} \mathrm{mL}^{-1}$ ). As shown in Fig. 5b, with the increase of the PTK 7 concentration, the fluorescence intensity at $580 \mathrm{~nm}$ gradually increased while the fluorescence intensity at $460 \mathrm{~nm}$ remained unchanged. To find out the relationship between the PTK 7 concentrations and changing fluorescence intensity, the concentrations of PTK 7 and ratiometric fluorescence intensity $\left(\mathrm{I}_{580} / \mathrm{I}_{460}\right)$ were analysed. As Fig. $5 \mathrm{c}$ shows, there was a good linear relationship between $\mathrm{I}_{580} / \mathrm{I}_{460}$ and the logarithm of PTK 7 concentrations ( $\log$ (PTK7)) when it was in the range of $0.1-100 \mathrm{ng} \mathrm{mL}^{-1}$. The calibration equation was $(\mathrm{n}=3)$ : $\mathrm{I}_{580} / \mathrm{I}_{460}=0.46653 \log (\mathrm{PTK} 7)+0.9226, \mathrm{r}=0.9922$. The limit of detection (LOD) based on $3 \sigma / \mathrm{K}$ (where $\sigma$ is the standard deviation of the blank measurement, and $\mathrm{k}$ is the slope of the calibration graph) was $0.016 \mathrm{ng} \mathrm{mL}^{-1}$, which was comparable or superior to most of the existing fluorescence probes for PTK 7 [43-46]. The comparison results are listed in Additional file 1: Table S1.

To ensure the selectivity, accuracy and repeatability of this developed probe, the relative studies were measured. The selectivity of this probe was measured by adding interfering proteins and ions, and the results are displayed in Fig. 6. As shown in Fig. 6a, the developed probe had a high response to PTK 7 and no response 

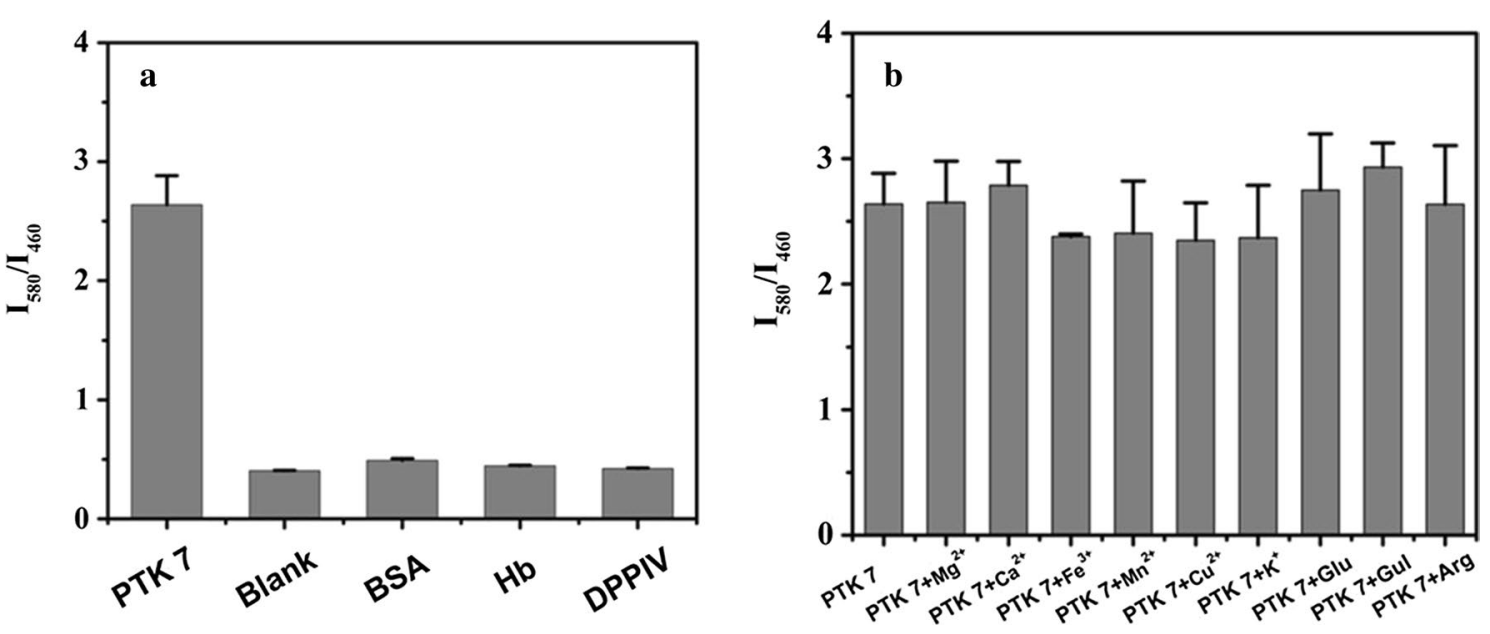

Fig. 6 Selectivity of PTK 7 detection, and concentration of PTK 7 was $100 \mathrm{ng} \mathrm{mL}^{-1}$. a Concentrations of BSA, Hb and DDPIV were $1000 \mathrm{ng} \mathrm{mL}^{-1}$, while $\mathbf{b}$ the concentrations of interferents $\left(\mathrm{Mg}^{2+}, \mathrm{Ca}^{2+}, \mathrm{Fe}^{3+}, \mathrm{Mn}^{2+}, \mathrm{Cu}^{2+}, \mathrm{K}^{+}, \mathrm{Glu}\right.$, Gul and Arg) were also $1000 \mathrm{ng} \mathrm{mL}^{-1}$

to other proteins (BSA, $\mathrm{Hb}$ and DPPIV). In addition, the probe showed no significant difference in its PTK 7 detection after the addition, or non-addition, of highconcentration interference ions (shown in Fig. 6b). These results indicate that the developed sensor has excellent selectivity and anti-interference mechanisms. Following this, the precision and accuracy of this ratiometric fluorescence probe were measured. As shown in Additional file 1: Table $S 2$, they were studied by assaying 0.20 , 5.00 and $80.00 \mathrm{ng} \mathrm{mL}^{-1}$ of PTK 7 in three separate runs. The intra-day and inter-day relative standard deviations (RSDs) were in the range of $3.6-10.2 \%$ and $1.8-10.9 \%$, respectively, while the accuracies were all in the range of $97.5-101.5 \%$. The results suggest that the ratiometric fluorescence probe has promising potential for the detection of PTK 7, and could be used for determination in real samples.

\section{Detection in real samples}

According to the functions of this developed probe, MCF-7 cells and human serum were used as the practical examples. Taking MCF-7 cell determination as an example, it could be seen that the intensity of the fluorescence of this developed probe increased with the rising cell density (Fig. 7a). Furthermore, it was observed in Fig. 7b that there was a good linear relationship between PTK 7 concentrations and MCF-7 cell density in the range of $1 \times 10^{4}$ to $5 \times 10^{5}$ per $\mathrm{mL}\left(\mathrm{C}_{\text {PTK } 7}=3.784 \mathrm{C}\right.$ cell density $+0.2126, r=0.9989, n=3$, and the unit of cell density was $1 \times 10^{4}$ per $\mathrm{mL}$ ). Next the recovery of PTK 7 during MCF-7 cell determination were evaluated, and the results are displayed in Additional file 1: Table S3. As shown, the recoveries for the detection of PTK 7 in MCF-7 cells were between $94.2 \%$ and $105.3 \%$. The RSDs were from 2.7 to $4.9 \%$. Additionally, the developed sensor was used to
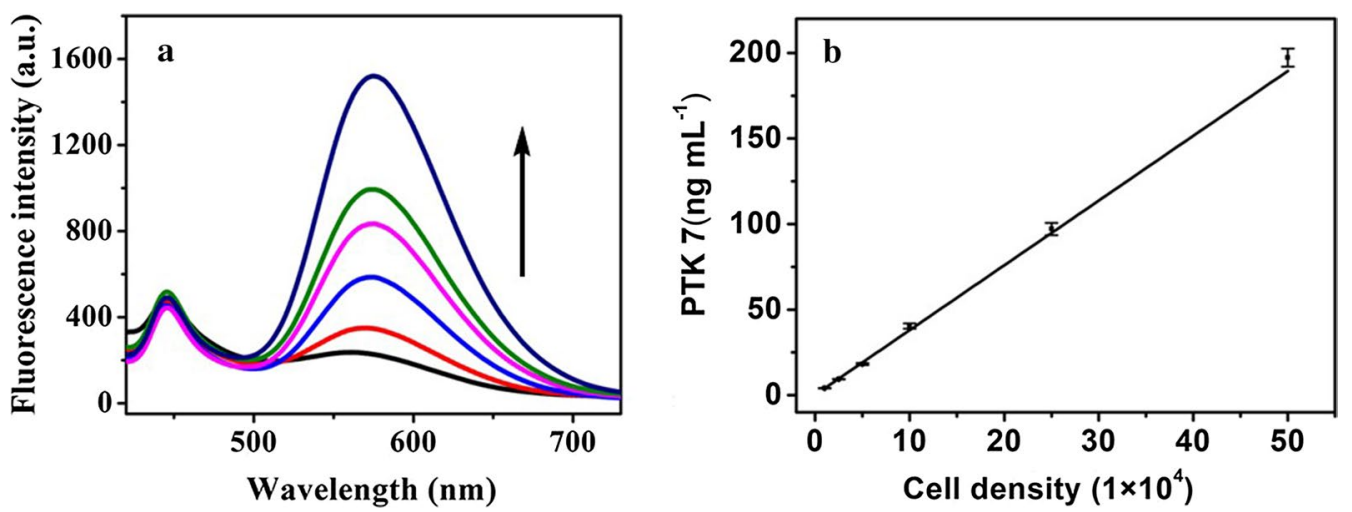

Fig. 7 a Fluorescence spectra of the probe in the presence of various cell densities of MCF-7 cells (in range of $1 \times 10^{4}$ to $5 \times 10^{5}$ per $\mathrm{mL}$ ). $\mathbf{b}$ The calibration curve of PTK 7 detection in MCF-7 cells in range of $1 \times 10^{4}-5 \times 10^{5}$ per mL 
detect PTK 7 in human serum, with the recovery results shown in Additional file 1: Table S4. Additional file 1: Table S4 shows that the PTK 7 recoveries in human serum were between 90.2 and $96.4 \%$ while the RSDs were from 3.9 to $6.7 \%$.

All of the results prove that this multi-nanomaterial based ratiometric fluorescence sensor has excellent sensitivity and selectivity, and that the probe could be used to detect PTK 7 in MCF-7 cells and human serum. These results indicate that the developed probe has great potential for use in clinical applications.

\section{Conclusion}

In summary, we successfully developed a ratiometric fluorescence probe based on multi-nanomaterials (b-CDs, $\mathrm{y}$-CDs and $\mathrm{Fe}_{3} \mathrm{O}_{4}$ MNPs) and an aptamer for PTK 7 detection in this work. In the absence of PTK 7, a y-CDs modified aptamer and cDNA modified $\mathrm{Fe}_{3} \mathrm{O}_{4}$ MNPs were assembled together, the fluorescence of $y$-CDs was quenched by $\mathrm{Fe}_{3} \mathrm{O}_{4}$ MNPs but recovered by adding PTK 7, and the ratiometric fluorescence probe was built by using b-CDs as the internal reference. Additionally, DNase I was added in order to set up a loop amplifier to enhance the sensitivity of this probe. This developed sensor exhibits a LOD of up to $0.016 \mathrm{ng} \mathrm{mL}^{-1}$, which outperforms previous methods and is highly sensitive and selective in its determination of PTK 7. Furthermore, this sensitive and selective probe was successfully used to detect PTK 7 in both MCF-7 cells and human serum. This thus shows a huge potential for its application in clinical practice.

\section{Supplementary Information}

The online version contains supplementary material available at https://doi. org/10.1186/s12951-021-00787-7.

Additional file 1: Figure $\mathrm{S} 1$. The zeta potential of $\mathrm{Fe}_{3} \mathrm{O}_{4} \mathrm{MNPS}$,

$\mathrm{Fe}_{3} \mathrm{O}_{4}-\mathrm{cDNA}$, $\mathrm{y}$-CDs and $\mathrm{y}$-CDs-APT. Figure S2. The optimum reactive conditions of PTK 7 determination. The temperature $(\mathbf{A})$ and the reactive time (B) of $\mathrm{Fe}_{3} \mathrm{O}_{4}$-CDNA and $\mathrm{y}$-CDs-APT hybridization reaction, the concentration $(\mathbf{C})$ and the reactive time $(\mathbf{D})$ of DNase I. All measurements were performed by single-factor test. Table S1. Comparison of the probe performance of this Work with those previously reported sensing methods. Table S2. Precision and accuracy of PTK 7 determination. Table S3. Recoveries for PTK 7 in MCF-7 cells $\left(5 \times 10^{4}\right)$ determination. Table S4. Recoveries for PTK 7 in human serum determination.

\section{Abbreviations}

PTK 7: Protein tyrosine kinase 7; CDs: Carbon dots; LOD: Limit of detection; MNPs: Magnetic nanoparticles; EDC: Diethylenetriamine, $\mathrm{N}$-(3-dimethylaminopropyl)-Nethylcarbodiimide hydrochloride; NHS: N-hydroxysuccinimide; GABA: 4-Aminobutyric acid; OPD: o-Phenylenediamine; TEM: Transmission electron microscopic images; RSDs: Relative standard deviations.

\section{Acknowledgements}

The serum samples were kindly supplied by Dr. Sun from Xuzhou First People's Hospital, and we would like to thank her for her help.

\section{Authors' contributions}

YM performed the data analyses and wrote the manuscript. YW performed the experiment. YL contributed significantly to analysis and manuscript preparation. LS helped perform the analysis with constructive discussions. DY contributed to the conception of the study. All authors read and approved the final manuscript.

\section{Funding}

National Science Foundation of China (61901405), the Project of Science and Technology of Xuzhou (KC19066), Excellent Talents Scientific Research Project (D2019026), Natural Science Foundation of Jiangsu Higher Education Institute of China (19KJD350003), Natural Science Foundation of Jiangsu Province, China (BK20201156) and China Postdoctoral Science Foundation (2020M671608).

\section{Availability of data and materials}

All data analyzed during this study are included in this published article.

Ethics approval and consent to participate

Not applicable.

\section{Consent for publication}

All authors of this work consent to the publication of the paper.

\section{Competing interests}

The authors declare that they have no known competing financial interests or personal relationships that could have appeared to influence the work reported in this paper.

Received: 12 December 2020 Accepted: 1 February 2021

Published online: 15 February 2021

\section{References}

1. Tang X, Deng R, Sun Y, Ren X, Zhou M, Li J. Amplified Tandem Spinachbased aptamer transcription enables low background miRNA detection. Anal Chem. 2018;90:10001-8.

2. Sahai N, Ahmad N, Gogoi M. Nanoparticles based drug delivery for tissue regeneration using biodegradable scaffolds: a review. Curr Pathobiol Rep. 2018;6:219-24.

3. Li K, Zhao X, Wei G, Su Z. Recent advances in the cancer bioimaging with graphene quantum dots. Curr Med Chem. 2018;25:2876-93.

4. Yang C-T, Pourhassan-Moghaddam M, Wu L, Bai P, Thierry B. Ultrasensitive detection of cancer prognostic miRNA biomarkers based on surface plasmon enhanced light scattering. ACS Sensors. 2017;2:635-40.

5. Wei W, Zhang X, Zhang S, Wei G, Su Z. Biomedical and bioactive engineered nanomaterials for targeted tumor photothermal therapy: a review. Mater Sci Eng, C. 2019;104:109891.

6. Zhang X, Liu W, Wang H, Zhao X, Zhang Z, Nienhaus GU, Shang L, Su Z. Self-assembled thermosensitive luminescent Nanoparticles with peptide-Au conjugates for cellular imaging and drug delivery. Chin Chem Lett. 2020;31(3):859-64.

7. Lecointre C, Simon V, Kerneur C, Allemand F, Fournet A, Montarras I, Pons J-L, Gelin M, Brignatz C, Urbach S, Labesse G, Roche S. Dimerization of the pragmin pseudo-kinase regulates protein tyrosine phosphorylation. Structure. 2018;26:545-54.

8. Jiao Y, Yin J, He H, Peng X, Gao Q, Duan C. Conformationally induced off-on cell membrane chemosensor targeting receptor protein-tyrosine kinases for in vivo and in vitro fluorescence of cancers. J Am Chem Soc. 2018;140:5882-5

9. Jiang C, Li Y, Liu C, Qiu L, Li Z. General and versatile fluorescence turn-on assay for detecting the activity of protein tyrosine kinases based on phosphorylation-inhibited tyrosyl oxidation. Chem Comm. 2016;52:12570-3.

10. Liang L, Lan F, Ge S, Yu J, Ren N, Yan M. Metal-enhanced ratiometric fluorescence/naked eye bimodal biosensor for lead ions analysis with bifunctional nanocomposite probes. Anal Chem. 2017;89:3597-605. 
11. Ahmad N, Bhatnagar S, Ali SS, Dutta R. Phytofabrication of bioinduced silver nanoparticles for biomedical applications. Int J Nanomed. 2015;10:7019-30.

12. Ahmad N, Bhatnagar S, Dubey SD, Saxena R, Sharma S, Dutta R. Nanopackaging in Food and Electron. 2017;24:45-97.

13. Ahmad N, Bhatnagar S, Saxena R, lqbal D, Ghosh AK, Dutta R. Biosynthesis and characterization of gold nanoparticles: kinetics, in vitro and in vivo study. Mater Sci Eng, C. 2017;78:553-64.

14. Khatoon A, Khan F, Ahmad N, Shaikh S, Rizvi SMD, Shakil S, Al-Qahtani MH, Abuzenadah AM, Tabrez S, Ahmed ABF, Alafnan A, Islam H, lqbal D, Dutta R. Silver nanoparticles from leaf extract of Mentha piperita: eco-friendly synthesis and effect on acetylcholinesterase activity. Life Sci. 2018;209:430-4.

15. Li K, Liu W, Ni Y, Li D, Lin D, Su Z, Wei G. Technical synthesis and biomedical applications of graphene quantum dots. J Mater Chem B. 2017:5:4811-26.

16. Zhao D, Zhang Z, Li C, Xiao X, Li J, Liu X, Cheng H. Yellow-emitting hydrophobic carbon dots via solid-phase synthesis and their applications. ACS Omega. 2020;5(35):22587-95.

17. Song Y, Zhu S, Xiang S, Zhao X, Zhang J, Zhang H, Fu Y, Yang B. Investigation into the fluorescence quenching behaviors and applications of carbon dots. Nanoscale. 2014;6:4676-82.

18. Xia Y, Wang L, Li J, Chen X, Lan J, Yan A, Lei Y, Yang S, Yang H, Chen J. A Ratiometric Fluorescent Bioprobe Based on Carbon Dots and Acridone Derivate for Signal Amplification Detection Exosomal microRNA. Anal Chem. 2018;90:8969-76.

19. Ma Y, Cen Y, Sohail M, Xu G, Wei F, Shi M, Xu X, Song Y, Ma Y, Hu Q. A ratiometric fluorescence universal platform based on N, Cu codoped carbon dots to detect metabolites participating in $\mathrm{H}_{2} \mathrm{O}_{2}$-generation reactions. ACS Appl Mater Interfaces. 2017;9:33011-9.

20. Cai Y, You J, You Z, Dong F, Du S, Zhang L. Profuse color-evolution-based fluorescent test paper sensor for rapid and visual monitoring of endogenous $\mathrm{Cu}(2+)$ in human urine. Biosens Bioelectron. 2018;99:332-7.

21. Kong R, Chen Z, Ye M, Zhang X, Tan W. Cell-SELEX-based aptamer-conjugated nanomaterials for enhanced targeting of cancer cells. Sci China Chem. 2011;54:1218-26.

22. Cheng X, Cen Y, Xu G, Wei F, Shi M, Xu X, Sohail M, Hu Q. Aptamer based fluorometric determination of ATP by exploiting the FRET between carbon dots and graphene oxide. Microch Acta. 2018;185(2):1-8.

23. Dong $\mathrm{H}$, Chen $\mathrm{H}$, Jiang J, Zhang $\mathrm{H}$, Cai C, Shen Q. Highly sensitive electrochemical detection of tumor exosomes based on aptamer recognitioninduced multi-DNA release and cyclic enzymatic amplification. Anal Chem. 2018;90:4507-13.

24. Shin W-R, Sekhon SS, Rhee S-K, Ko JH, Ahn J-Y, Min J, Kim Y-H. Aptamerbased paper strip sensor for detecting Vibrio fischeri. ACS Comb. Sci. 2018;20:261-8

25. Song $Y, X u$ G, Wei F, Cen Y, Sohail M, Shi M, Xu X, Ma Y, Ma Y, Hu Q. Aptamer-based fluorescent platform for ultrasensitive adenosine detection utilizing Fe3O4 magnetic nanoparticles and silver nanoparticles. Microchim Acta. 2018;185:1-9.

26. Yao Q, Wang Y, Wang J, Chen S, Liu H, Jiang Z, Zhang X, Liu S, Yuan Q, Zhou X. An ultrasensitive diagnostic biochip based on biomimetic periodic nanostructure-assisted rolling circle amplification. ACS Nano. 2018;12:6777-83.

27. Xu S, Nie Y, Jiang L, Wang J, Xu G, Wang W, Luo X. Polydopamine nanosphere/gold nanocluster (Au NC)-based Nanoplatform for dual color simultaneous detection of multiple tumor-related microRNAs with DNase-l-assisted target recycling amplification. Anal Chem. 2018;90:4039-45.

28. Jiao Y, Gao Y, Meng Y, Lu W, Liu Y, Han H, Shuang S, Li L, Dong C. One-step synthesis of label-free ratiometric fluorescence carbon dots for the detection of silver ions and glutathione and cellular imaging applications. ACS Appl Mater Interfaces. 2019;11(18):16822-9.

29. Ahmed $\mathrm{SR}$, Cirone J, Chen A. Fluorescent Fe3O4 Quantum Dots for $\mathrm{H} 2 \mathrm{O} 2$ Detection. ACS Appl. Nano Mater. 2019;2:2076-85.
30. Kwon OS, Song HS, Park TH, Jang J. Conducting nanomaterial sensor using natural receptors. Chem Rev. 2019;119:36-93.

31. Wu Y, Ma Y, Xu G, Wei F, Ma Y, Song Q, Wang X, Tang T, Song Y, Shi M, Xu X, Hu Q. Metal-organic framework coated Fe3O4 magnetic nanoparticles with peroxidase-like activity for colorimetric sensing of cholesterol. Sensor Actuat B-Chem. 2017;249:195-202.

32. Shi M, Cen Y, Sohail M, Xu G, Wei F, Ma Y, Xu X, Ma Y, Song Y, Hu Q. Aptamer based fluorometric beta-lactoglobulin assay based on the use of magnetic nanoparticles and carbon dots. Microchim Acta. 2018;85(1):1-8

33. Ma Y, Song Y, Ma Y, Wei F, Xu G, Cen Y, Shi M, Xu X, Hu Q. N-doped carbon dots as a fluorescent probe for the sensitive and facile detection of carbamazepine based on the inner filter effect. New J Chem. 2018;42:8992-7.

34. Lu W, Jiao Y, Gao Y, Qiao J, Mozneb M, Shuang S, Dong C, Li C. Bright yellow fluorescent carbon dots as a multifunctional sensing platform for the label-free detection of fluoroquinolones and histidine. ACS Appl Mate Interfaces. 2018;10:42915-24.

35. Song $Q$, Ma Y, Wang $X$, Tang T, Song Y, Ma Y, Xu G, Wei F, Cen Y, Hu Q. "Onoff-on" Fluorescent System for Detection of Zn2 + in Biological Samples Using Quantum Dots-carbon Dots Ratiometric Nanosensor. J Colloid Interf. Sci. 2018;516:522-8.

36. Ma Y, Xu G, Wei F, Cen Y, Song Y, Ma Y, Xu X, Shi M, Sohail M, Hu Q. Carbon dots based immunosorbent assay for the determination of GFAP in human serum. Nanotechnology. 2018;29:145501-9.

37. Wu L, Wu IC, DuFort CC, Carlson MA, Wu X, Chen L, Kuo CT, Qin Y, Yu J, Hingorani SR, Chiu DT. Photostable ratiometric Pdot probe for in vitro and in vivo imaging of hypochlorous acid. J Am Chem Soc. 2017;139:6911-8.

38. Song Y, Tang T, Wang X, Xu G, Wei F, Wu Y, Song Q, Ma Y, Ma Y, Cen Y, Hu Q. Highly selective and sensitive detection of adenosine utilizing signal amplification based on silver ions-assisted cation exchange reaction with Cdte quantum dots. Sensor Actuat B-Chem. 2017;247:305-11.

39. Zhang Q, Wang F, Zhang H, Zhang Y, Liu M, Liu Y. Universal Ti3C2 MXenes based self-standard ratiometric fluorescence resonance energy transfer platform for highly sensitive detection of exosomes. Anal Chem. 2018;90:12737-44.

40. Peng C, Xing H, Fan X, Xue Y, Li J, Wang E. Glutathione regulated inner filter effect of $\mathrm{MnO}_{2}$ nanosheets on boron nitride quantum dots for sensitive assay. Anal Chem. 2019;91:5762-7.

41. Pritzl SD, Pschunder F, Ehrat F, Bhattacharyya S, Lohmüller T, Huergo MA, Feldmann J. Trans-membrane fluorescence enhancement by carbon dots: Ionic interactions and energy transfer. Nano Lett. 2019;19(6):3886-91.

42. Liu Y, Hu J, Li Y, Wei H-P, Li X-S, Zhang X-H, Chen S-M, Chen X-Q. Synthesis of Dolyethyleneimine capped carbon dots for preconcentration and slurry sampling analysis of trace chromium in environmental water samples. Talanta. 2015;134:16-23.

43. Wang Y-M, Wu Z, Liu S-J, Chu X. Structure-switching aptamer triggering hybridization chain reaction on the cell surface for activatable theranostics. Anal Chem. 2015;87:6470-4.

44. Miao X, Li Z, Zhu A, Feng Z, Tian J, Peng X. Ultrasensitive Electrochemical Detection of Protein Tyrosine Kinase- 7 by Gold Nanoparticles and Methylene Blue Assisted Signal Amplification. Biosens Bioelectron. 2016:83:39-44

45. Lin S, Gao W, Tian Z, Yang C, Lu L, Mergny J-L, Leung C-H, Ma D-L. Luminescence switch-on detection of protein tyrosine kinase-7 using a G-Quadruplex-selective Probe. Chem Sci. 2015;6:4284-90.

46. Liu Z, Chen W, Han Y, Ouyang J, Chen M, Hu S, Deng L, Liu Y-N. A label-free sensitive method for membrane protein detection based on aptamer and AgNCs transfer. Talanta. 2017;175:470-6.

\section{Publisher's Note}

Springer Nature remains neutral with regard to jurisdictional claims in published maps and institutional affiliations. 